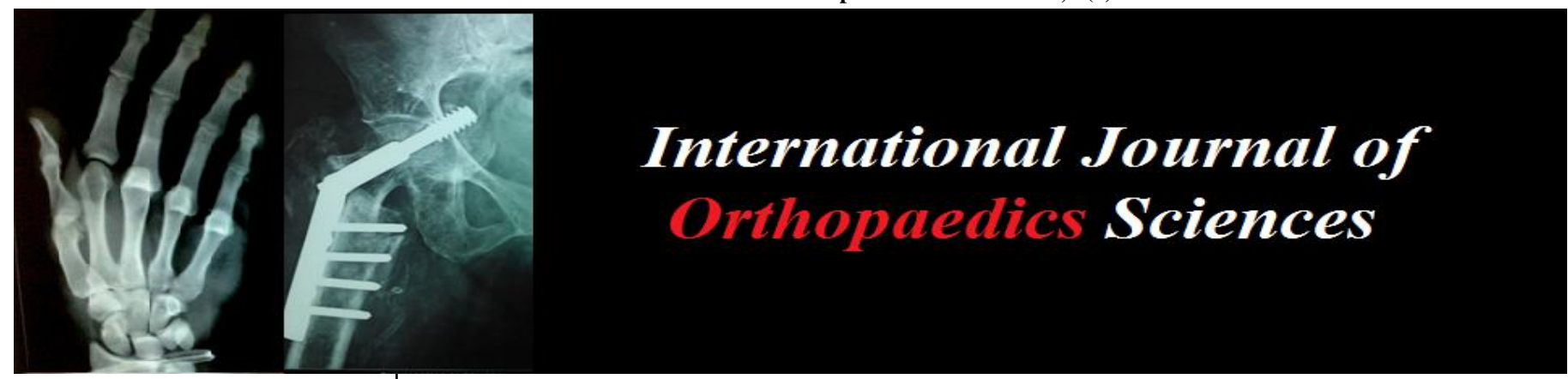

ISSN: $2395-1958$

IJOS 2019; 5(2): 202-206

(C) 2019 IJOS

www.orthopaper.com

Received: 18-02-2019

Accepted: 19-03-2019

Dr. Vipul kuvad

Assistant. Professor.

Orthopedics GMERS Medical,

College Sola, Gujarat, India

Dr. Somesh Singh

Associate Professor, Orthopedics

GMERS Medical, College Sola,

Gujarat, India
Correspondence

Dr. Vipul kuvad

Assistant. Professor.

Orthopedics GMERS Medical,

College Sola, Gujarat, India

\section{A study to assess the effect of articular congruency and displacement in treatment of fracture of lateral condyle of humerus in children}

\section{Dr. Vipul Kuvad and Dr. Somesh Singh}

DOI: https://doi.org/10.22271/ortho.2019.v5.i2d.39

\section{Abstract}

Background: Lateral condyle fractures of the distal humerus are the second most common fractures at the elbow in the paediatric population which if not properly treated leads to non-union deformity and other complications. Operative intervention with closed reduction and percutaneous pinning or open reduction internal fixation are indicated for a malaligned articular surface and/or an unstable fracture. Intraoperative arthrograms helps in doubtful congruency of joint surface and in assessing joint reduction. We present our results of treatment of fracture lateral condyle humerus in 24 patients and effect of the fracture displacement and congruency on functional and clinical outcome.

Aim: The aim of our study was to assess functional and clinical results of fracture lateral condyle of humerus in children based on articular congruency.

Materials and Methods: It was a retrospective study of total 24 patient with isolated fracture of lateral condyle of humerus. Open fracture and other co morbidities cases were excluded. Functional and clinical outcome according to hardware criteria was evaluated.

Results: There were 24 cases in study with average age of 7.54 years. There were 19 male and 05 female. According to Jacob classification 06 were type I, 06 were type II and 12 were type III. Arthrography helped in decision making in doubtful cases of joint congruency. $100 \%$ excellent results achieved in type I and type II fractures whereas in $85 \%$ cases of type III fractures achieved excellent to good results. Increase operative time is associated with spur formation.

Conclusion: Appropriate plan of treatment decided on articular congruency and displacement with the help of arthrography gives excellent results in fracture lateral condyle of humerus.

Keywords: Lateral condyle humerus, pediatric humerus fracture, Arthrography elbow

\section{Introduction}

Lateral condyle fractures of the distal humerus are the second most common fractures at the elbow in the paediatric population usually between the ages of 6-10 years. The diagnosis can be difficult both radiologically and clinically due to extension into the articular surface which if not treated properly leads to complications like non-union, mal union, fish tail deformity ${ }^{[1]}$ These fractures need perfect anatomical reduction and articular alignment to prevent complications.

Most of the fractures occur between 4 to10 years of age with peak incidence at 5-6 year age ${ }^{\text {[2] }}$ Controversy exists regarding the management of non-displaced and minimally displaced (less than $2 \mathrm{~mm}$ ) fractures. These fractures account for $33 \%$ to $69 \%$ of lateral humeral condylar fractures ${ }^{[3-5]}$. It is generally accepted that open reduction of the fracture and internal fixation with screws or wires constitutes the method of choice.

The Milch classification is widely used, and they are type I and type II according to whether the fracture exited through the capitellar-trochlear groove or through the trochlear, respectively ${ }^{[6]}$ The Jacob classification dictates whether surgical intervention is required. A Jacob I is non-displaced, II is displaced by $2 \mathrm{~mm}$, but not malrotated. Type III is displacement with malrotation. The aim of lateral humeral condyle fracture treatment is to ensure healing of the fracture and to prevent pseudoarthrosis, malunion, deformities and functional disorders [7] Traditionally, undisplaced stable fractures were treated in cast immobilisation with observation. 
Articular fractures that have a hinge may be treated with closed reduction and percutaneous pinning. In certain situations, an arthrogram or an MRI scan may help define articular congruity and adequacy of the reduction [7, 8] Fractures that are unstable, malrotated and displaced by over $2 \mathrm{~mm}$ usually undergo open reduction internal fixation usually with wires, smooth pins or screws.

Though recent methods of diagnosis and proper understanding of anatomy and treatment have reduced complications, there is constant need of improvement for early diagnosis and adequate management which may not be too much demanding. We have tried to study proper classification and the treatment in this study.

\section{Materials and Methods}

Retrospective study of 24 cases of lateral condyle fractures treated was performed. Initial assessment of the patients was performed in the Emergency Department of our Institution. The injured limb was examined for deformity, wounds and neurovascular integrity. Antero-posterior, oblique and lateral radiographs of the elbow were routinely performed.

Exclusion criteria involved: a) those with open fractures; b) those whose injury involved more than the lateral condyle, and/or those with a dislocation and d) those who underwent fixation more than two weeks after the injury.

All fractures were classified by the following system: A Type I fracture is displaced less than $2 \mathrm{~mm}$. In a Type II fracture there is $>2 \mathrm{~mm}$ of displacement with intact articular cartilage, as demonstrated by arthrogram. In a Type III fracture there is $>2 \mathrm{~mm}$ of displacement and the articular surface is not intact.

The acceptable displacement for conservative management in an above elbow plaster of Paris (POP) cast was up to $2 \mathrm{~mm}$. Patients who were treated conservatively were closely followed up with radiographs every week to ensure that the fracture has not displaced. The POP cast was removed upon radiological union typically between 4 and 6 weeks-and physiotherapy commenced.

The surgical technique is as follows: First, fracture displacement was evaluated. If there was uncertainty as to the congruity of the articular surface, an arthrogram was performed. If the articular surface was congruent, then closed reduction and pinning were performed. If the articular surface was not congruent, an open reduction and internal fixation was performed. The fracture was identified and reduced via a dorsolateral approach to the distal humerus, through the interval between brachioradialis and triceps. The articular surface was directly visualized and reduced, and either 2 or 3 $\mathrm{k}$-wires were placed in a divergent pattern to stabilize the fracture. Subsequently, an above elbow POP in neutral position was applied. Fluoroscopy was used intraoperatively to help assess fracture reduction and pin placement. Postoperatively, the first radiographic assessment was at 1 week after surgery to assure that the fracture reduction was maintained.

All patients were then monitored until they showed solid radiographic healing, regained their motion, became asymptomatic and had no residual problems.

Follow up evaluation was done using functional and overall grading and Hardacre ${ }^{[9]}$ criteria. Hardacre Criteria considers functional and cosmetic base which was used for the evaluation of our results. Excellent result means no loss of motion, no alteration in carrying angle, and no symptoms. Good result is characterized by a satisfactory functional range of motion, lacking no more than 15 degrees of complete extension with no arthritic or neurological symptoms. Poor results included disabling loss of motion, conspicuous alterations of carrying angle, arthritic symptoms, ulnar neuritis, and radiographic findings of nonunion or avascular necrosis.

\section{Results}

We have studied 24 cases of fracture lateral condyle of humerus children at our hospital and make following observations regarding various factors affecting fracture \& plan of management and results of various fracture type.

Table 1: Age group distribution

\begin{tabular}{|c|c|c|}
\hline Age & Total & \% \\
\hline $2-5$ & 03 & 12.5 \\
\hline $6-9$ & 15 & 62.5 \\
\hline $10-13$ & 06 & 25 \\
\hline Sex & Total & $\%$ \\
\hline Male & 19 & 79 \\
\hline Female & 05 & 21 \\
\hline Milch type & Total & \% \\
\hline Type-I & 07 & 29 \\
\hline Type-II & 17 & 71 \\
\hline Jacob Type of fracture & Total & $\%$ \\
\hline Type-I & 6 & 25 \\
\hline Type-II & 6 & 25 \\
\hline Type-III & 12 & 50 \\
\hline
\end{tabular}

6-9 age group is commonly affected. Male are commonly affected than female in ratio of $4: 1$

Milch type II is very common. We have not followed this classification for treatment.

Table 2: Treatment modality according to fracture type

\begin{tabular}{|c|c|c|c|c|}
\hline \multirow{2}{*}{ Type of fracture } & \multirow{2}{*}{ Total } & \multirow{2}{*}{ Conservative } & \multicolumn{2}{|c|}{ Operative } \\
\cline { 4 - 5 } & & & Close & Open \\
\hline Type-I & 6 & 6 & - & - \\
\hline Type-II & 6 & 1 & 3 & 2 \\
\hline Type-III & 12 & - & - & 12 \\
\hline
\end{tabular}

Table 3: Difference in carrying angle of normal and affected side according to type of fracture

\begin{tabular}{|c|c|c|c|}
\hline Difference & Type-I & Type-II & Type-III \\
\hline $0-5^{0}$ & 6 & 6 & 9 \\
\hline $6-10^{0}$ & 0 & 0 & 2 \\
\hline$>10^{0}$ & 0 & 0 & 1 \\
\hline
\end{tabular}

Table 4: Complications and their correlation with results

\begin{tabular}{|c|c|c|c|c|}
\hline Complications & Total & & Results & \\
\hline & & Excellent & Good & Poor \\
\hline Loss of movement & 3 & 0 & 1 & 2 \\
\hline Altered carrying angle & 3 & 0 & 2 & 1 \\
\hline Spur formation & 6 & 4 & 0 & 2 \\
\hline Pin tract infection & 1 & 1 & 0 & 0 \\
\hline Delayed union & 2 & 0 & 0 & 2 \\
\hline Non-union & 0 & 0 & 0 & 0 \\
\hline
\end{tabular}

Table 5: Correlation between operative times and spur formation

\begin{tabular}{|c|c|c|}
\hline Time (minutes) & Total & \% \\
\hline$<30$ & 1 & 17 \\
\hline $30-60$ & 1 & 17 \\
\hline$>60$ & 4 & 66 \\
\hline
\end{tabular}


Table 6: Results according to treatment modality

\begin{tabular}{|c|c|c|c|}
\hline \multirow{2}{*}{ Results } & \multirow{2}{*}{ Conservative } & \multicolumn{2}{|c|}{ Operative } \\
\cline { 3 - 4 } & & Close & Open \\
\hline Excellent & $7(100 \%)$ & $3(100 \%)$ & $9(64 \%)$ \\
\hline Good & 0 & 0 & $3(21 \%)$ \\
\hline Poor & 0 & 0 & $2(15 \%)$ \\
\hline
\end{tabular}

In 2 cases of poor results one had full range of motion but progressive valgus deformity while other one had disabling loss of motion but inconspicuous carrying angle.

Table 7: Results according to type of fracture

\begin{tabular}{|c|c|c|c|}
\hline Results/type of fracture & Type-I & Type-II & Type-III \\
\hline Excellent & $6(100 \%)$ & $6(100 \%)$ & $7(59 \%)$ \\
\hline Good & 0 & 0 & $3(24 \%)$ \\
\hline Poor & 0 & 0 & $2(17 \%)$ \\
\hline
\end{tabular}

\section{Discussion}

This is a study of 24 cases of fracture of lateral condyle of humerus treated at our hospital. This study is mainly for various factors affecting different type of fracture, plan of management \& results of various fracture type. In our study majority of patient are between 6-9 year age group and minimum $\&$ maximum being $4 \& 13$ years respectively. There is preponderance of male. Left side elbow is involved more than right. Most of the cases (77\%) non-dominant hand is involved. Average hospital stay is of 2.5 days.

Fall on outstretch hand with elbow extended is the commonest mode of injury, which supports varus strain theory. $17 \%$ cases had history of fall on flexed elbow. One case is of direct injury, which had undisplaced fracture lateral condyle.

There is a group of minimally displaced fracture that do not necessarily require open reduction $\&$ internal fixation. Some fractures have congruent joint surface with a cartilage hinge that holds the fragment attached to distal humerus. This configuration is relatively stable and can be treated by close reduction and percutaneous pinning. In our study we found that proper understanding of articular congruency on xray and arthrography $[10,11]$ helped us to treat these patient by close means and get excellent results. This treatment modality avoid operative complications like avascular necrosis and nonunion which results from excessive soft tissue dissection.

In 14 cases of open reduction, on perop findings we observe that none of the fracture appeared to traverse the ossified portion of the capitellum (Milch type I). On X-ray 4 out of this 14 cases were of Milch type I, but on perop examination they were found to be Milch type II. So, intraoperative findings did not correlate with the preoperative radiographic diagnosis. 3 out of 7 cases of undisplaced fracture (type I) had Milch type II fracture on X-ray. Generally Milch type II fractures are treated by open reduction but we treated this patients by conservative means and got excellent results. Milch classification does not provide useful clinical information for plan of management. So we have classified this fracture according to displacement. We agree with the work of Mirsky and Karas ${ }^{[12]}$ in which they observed the same findings.

$\mathrm{K}$-wire is the most reliable implant for treating lateral condyle fractures as threaded screw or compressive plate can cause absorption of epiphysis and later on valgus and fishtail deformity. In some cases one transverse K-wire through physis is helpful to prevent rotation. $2 \mathrm{~mm}$ is proper size for fixing these fractures. There is definite association between operative time and spur formation as we found in 4 cases. More operative time causes more soft tissue dissection and fragment manipulation, which leads to periosteal stripping and spur formation.

Usual time for union of fracture of lateral condyle is 6 week. Implant removal should be considered at that time. In one case, early removal of $\mathrm{K}$-wire at 4-weeks resulted in delayed union. Same findings are observed by Cardona and Riddleo. [13] Although physiotherapy was started earlier in operative procedure it had to be given for longer duration as compared to conservative treatment. Postoperative fibrosis may be responsible for this. In operative procedure we can start physiotherapy as early as possible from 4-weeks. This reduces elbow stiffness and number of visits. In physiotherapy schedule we found that elbow flexion is achieved earlier than the extension.

All (3) cases of loss of movement are associated with type III fracture. Although in one case carrying angle is affected by $15^{\circ}$, movement is full. This patient had progressive cubitus valgus deformity without any affection of ulnar nerve at the time of final follow-up of 2 year. In all cases (14) of open reduction there is about $3-4^{\circ}$ loss of hyperextension compared to normal side. All cases (4) of various deformities are also associated with type III fracture.

Lateral condyle overgrowth is noted in 10 out of 14 cases of open reduction, which has no significance functionally except parent's concern so parents should be explained about this bony bump before surgery. Poor results also have complications but their final range of motion is good compared to pre-operative movement. Average increase in range of motion of these cases is of $80^{\circ}$. Complications like pin tract infection and spur formation are also noted but they did not alter the final outcome. There is not a single case of non-union but there are 2 cases of delayed union.

Results are evaluated according Hardacre criteria. We have modified the criteria for the carrrying angle. Minimal alteration (0-5), alteration is inconspicuous $\left(6-10^{\circ}\right)$ and alteration in carrying angle is conspicuous $\left(10^{\circ}\right)$. Minimal to inconspicuous alteration in carrying angle did not alter the range of motion, which is functional requirement of the patient. All fresh cases have excellent results with specific treatment modality according to type of fracture.

\section{Conclusion}

At final, we conclude that depending on displacement of fracture appropriate treatment should be given and minimally displaced fracture with articular congruency should be fixed with percutaneous $\mathrm{K}$-wiring and thus reducing complications and operative burden.

\section{Conflict of Interest}

The authors declare no conflict of interest. 


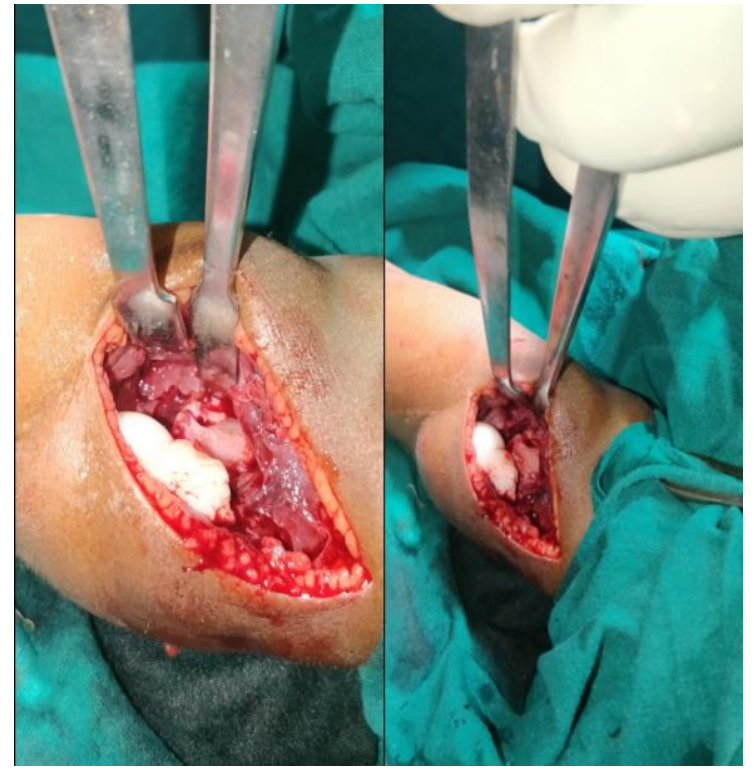

Fig 1: The joint surface was accurately reduced with minimal dissection of soft tissues from the distal fragment in order to reduce the risk of avascular necrosis of the capitellum.

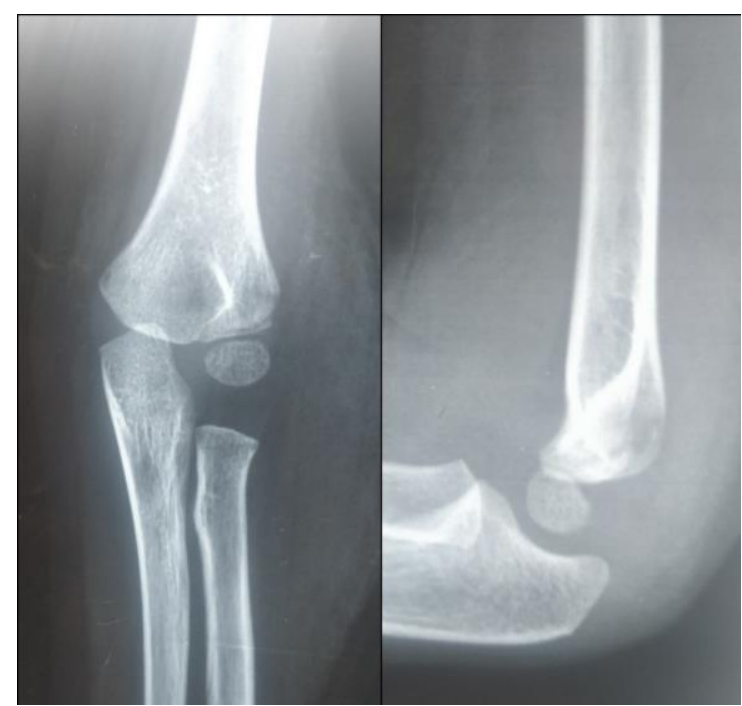

Fig 2: Fracture is classified on the basis of displacement on X-ray.

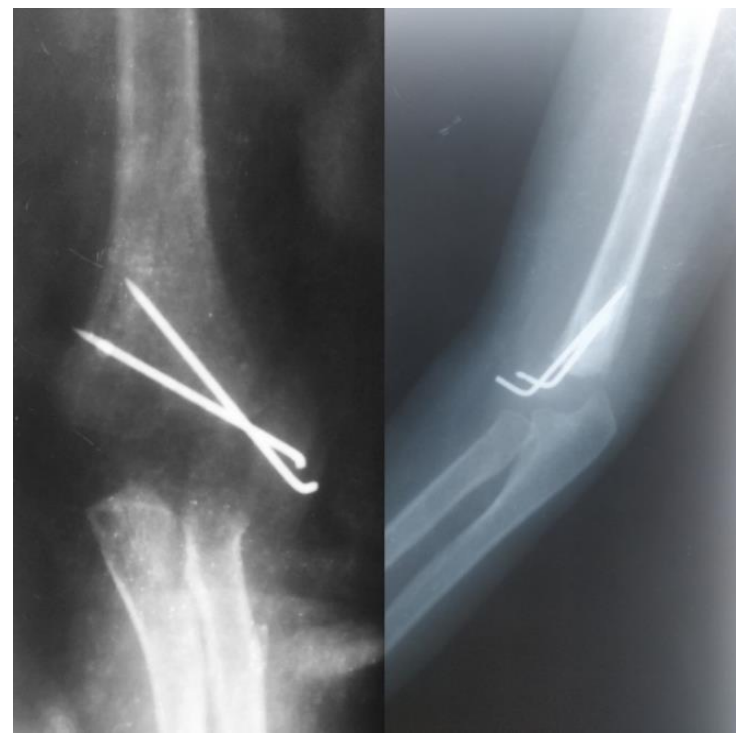

Fig 3: Undisplaced, minimally displaced and totally displaced.

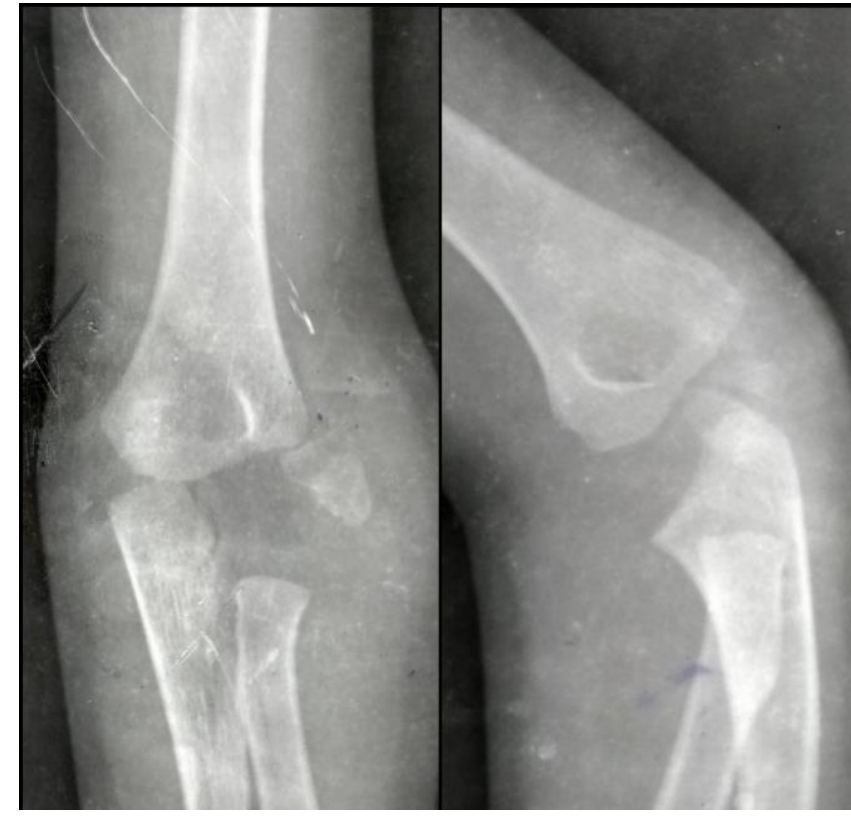

Fig 4: In case of doubt on displacement or on articular congruency arthrography was done

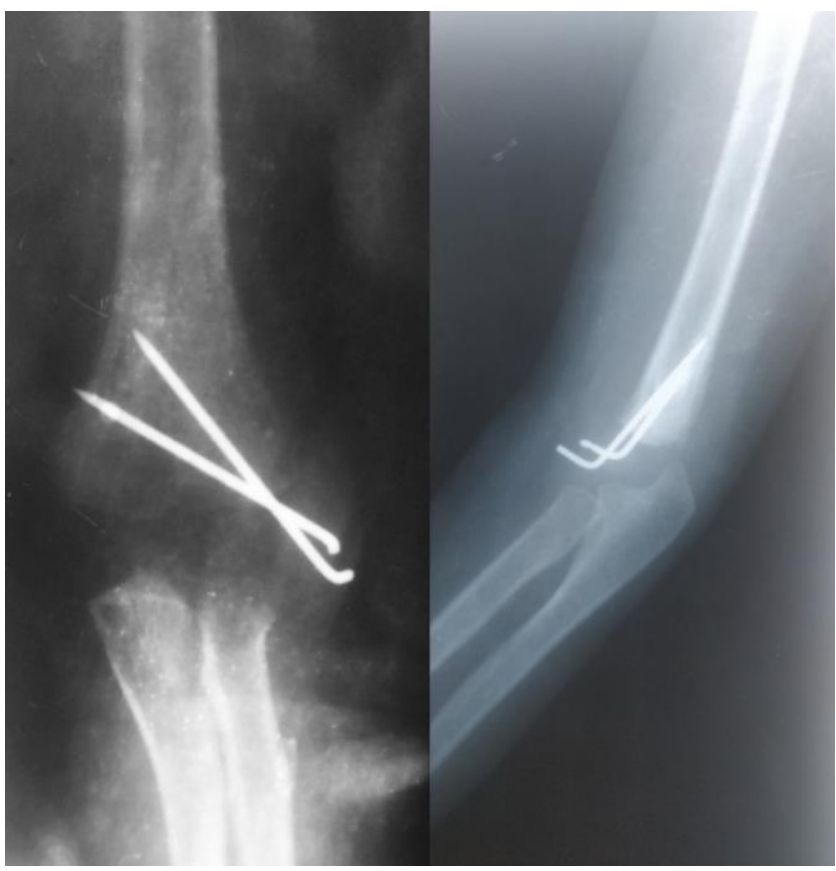

Fig 5: Arthrography was able to show the status of articular congruency

\section{Reference}

1. Jakob R, Fowles JV, Rang M, Kassab MT. Observations concerning fractures of the lateral humeral condyle in children. J Bone Joint Surg Br. 1975; 57(4):430-6.

2. dePablos J, Tejero A. Fractures of the upper limb and hand. In: Benson MK, Fixen JA, Macnicol MF, eds. Children's Orthopaedics and Fractures. 2nd edn. New York: Churchill Livingston, 2002, 609-32

3. Rutherford A: Fractures of the lateral humeral condyle in children. J Bone Joint Surg Am. 1985; 67(6):851-856.

4. Foster DE, Sullivan JA, Gross RH. Lateral humeral condylar fractures in children. J Pediatr Orthop. 1985; 5(1):16-22.

5. Pirker ME, Weinberg AM, Höllwarth ME, Haberlik A. Subsequent displacement of initially nondisplaced and minimally displaced fractures of the lateral humeral condyle in children. J Trauma. 2005; 58(6):1202-1207. 
6. Milch H. Fractures and fracture dislocations of the humeral condyles. J Trauma. 1964; 4:592-607.

7. Rockwood CA, Wilkins KE, Beaty JH, Kasser JR. Rockwood and Wilkins' fractures in children, 6th edn. Lippincott Williams \& Wilkins, Philadelphia, 2006; Xv: 1200

8. Beltran J, Rosenberg ZS. MR imaging of pediatric elbow fractures. Magn Reson Imaging Clin N Am. 1997; 5(3):567-578

9. Hardacre JA, Nahigian SH, Froimson AI, Brown JE. J Bone Joint Surg Am. 1971; 53(6):1083-95

10. Marzo John M. d'Amato, Charles, Strong, Munro, Gillespie, Robert M.D. Journal of Pediatric Orthopaedics, 1990.

11. Yates C, Sullivan JA. Arthrographic diagnosis of elbow injuries in children. J Pediatr Orthop 1987; 7:54-60.

12. Mirsky EC, Karas EH, Weiner LS. Lateral condyle fractures in children: Evaluation of classification and treatment. J Orthop Trauma. 1997; 11(2):117-120.

13. Cardona JI, Riddle E, Kumar SJ. J Pediatr Orthop. 2002; 22(2):194-7 